\title{
Upregulation of SNTB1 Correlates with Poor Prognosis and Promotes Cell Growth in Colorectal Cancer
}

\section{Liya Liu}

Fujian University of Traditional Chinese Medicine

\section{Youqin Chen}

Case Western Reserve University School of Medicine

\section{Xiaoying Lin}

Fujian University of Traditional Chinese Medicine

\section{Meizhu Wu}

Fujian University of Traditional Chinese Medicine

\section{Jiapeng Li}

Fujian University of Traditional Chinese Medicine

\section{Qiurong Xie}

Fujian University of Traditional Chinese Medicine

\section{Thomas Joseph Sferra}

Case Western Reserve University School of Medicine

\section{Yuying Han}

Fujian University of Traditional Chinese Medicine

Huixin Liu

Fujian University of Traditional Chinese Medicine

\section{Liujing Cao}

Fujian University of Traditional Chinese Medicine

\section{Mengying Yao}

Fujian University of Traditional Chinese Medicine

\section{Jun Peng}

Fujian University of Traditional Chinese Medicine

Aling Shen ( $\sim 2018025 @ f j t c m . e d u . c n)$

Fujian University of Traditional Chinese Medicine https://orcid.org/0000-0002-5081-3523

\section{Primary research}

Keywords: Colorectal cancer, syntrophin beta 1, growth, overall survival, Protein kinase N2 
Posted Date: June 11th, 2021

DOI: https://doi.org/10.21203/rs.3.rs-549083/v1

License: (c) (i) This work is licensed under a Creative Commons Attribution 4.0 International License. Read Full License 


\section{Abstract}

Background: Colorectal cancer (CRC) is one of the most highly malignant tumors and has a complicated pathogenesis. A preliminary study identified syntrophin beta 1 (SNTB1) as a potential oncogene in CRC. However, the clinical significance, biological function, and underlying mechanisms of SNTB1 in CRC are unknown. Thus, the present study aimed to investigate the function of SNTB1 in CRC.

Methods: The expression profile of SNTB1 in CRC samples was evaluated by database analysis, cDNA array, tissue microarray, Quantitative real-time PCR (qPCR), and immunohistochemistry. SNTB1 expression in human CRC cells was silenced using short hairpin RNAs and its mRNA and protein levels were assessed by qPCR and western blotting, respectively. Cell proliferation, colony formation, cell cycle and apoptosis were determined by the cell counting, colony formation, and flow cytometry assays, respectively. A xenograft nude mouse model of CRC was established for validating the roles of SNTB1 in vivo. Immunohistochemistry was used to score the expression of SNTB1 in tissue samples. The isobaric tags for relative and absolute quantification (iTRAQ) was used to analyze the differentially expressed proteins after knockdown of SNTB1 in CRC cells.

Results: SNTB1 expression was increased in CRC tissue compared with adjacent noncancerous tissues and the increased expression was associated with shorter overall survival of CRC patients. Silencing of SNTB1 suppressed cell viability and survival, induced cell cycle arrest and apoptosis in vitro, and inhibited the growth of CRC cells in vivo. Further elucidation of the regulation of STNB on CRC growth by iTRAQ analysis identified 210 up-regulated and 55 down-regulated proteins in CRC cells after SNTB knockdown. A PPI network analysis identified protein kinase N2 (PKN2) as a hub protein and was upregulated in CRC cells after SNTB1 knockdown. Western-blot analysis further confirmed that SNTB1 knockdown significantly up-regulated PKN2 protein expression in CRC cells and decreased the phosphorylation of both ERK1/2 and AKT.

Conclusion: These findings indicate that SNTB1 is overexpressed in CRC. Elevated SNTB1 levels are correlated with shorter patient survival. Importantly, SNTB1 promoted tumor growth and progression of CRC, possibly by reducing the expression of PKN2 and activating the ERK and AKT signaling pathway. Our study highlights the potential of SNTB1 as a new prognostic predictor and therapeutic target for CRC.

\section{Background}

Colorectal cancer (CRC) is the third most frequently diagnosed cancer (6.1\% of all cancers) and the second leading cause of cancer death (9.2\% of all cancer deaths) [1]. Although great strides have been made in the treatment of $\mathrm{CRC}$, metastasis, recurrence, and chemoresistance remain major obstacles to effective treatment in all patients [2-4]. Therefore, obtaining a better understanding of the molecular mechanisms responsible for the rapid growth and tumorigenic properties of CRC may lead to novel therapeutic strategies for this disease. 
In preliminary published work, using a gene expression profile microarray to screen for differential expressed genes (DEGs) between paired CRC and noncancerous tissue, we found the level of syntrophin beta 1 (SNTB1) to be upregulated in CRC tissue (GEO ID: GSE113513). However, the clinical significance, biological function, and underlying regulating mechanisms of SNTB1 remain largely unknown in most of cancers, including CRC, which encouraged us to further explore the role of SNTB1 in CRC.

SNTB1 is a member of syntrophin gene family which consists of five homologous isoforms, $\alpha 1, \beta 1, \beta 2, \gamma 1$ and $\mathrm{2} 2[5-7]$. Two pleckstrin homology $(\mathrm{PH})$ domains, a PDZ domain, and a conserved syntrophin unique (SU) region constitute the structure of SNTB1 and are sites of interaction with other proteins [8]. SNTB1 is associated with dystrophin and dystrophin-related proteins, which is one of the members of syntrophin gene family and mainly expressed in skeletal and smooth muscle, liver and kidney and expressed at low levels in many other tissues, including colorectal tissue [5]. Loss and reduction of SNTB1 is closely associated with Duchenne muscular dystrophy and Becker's muscular dystrophy [6]. The functional variants of SNTB1 can cause other abnormalities such as acute pancreatitis, oral cancer and severe myopia $[7,9,10]$. Whether SNTB1 has a pathological role in CRC is unknown.

In the present study, bioinformatics analysis of online databases, cDNA array, and tissue microarray (TMA) were used to evaluate the expression profile of SNTB1 and the relationship between SNTB1 expression and clinical pathological parameters in CRC. Moreover, a series of in vitro and in vivo experiments using representative CRC cell lines and xenograft nude mice models were conducted to examine the functional role of SNTB1.Furthermore, the underlying mechanisms of SNTB1 on tumor growth were explored.

\section{Methods}

\section{Materials and reagents}

The antibodies against SNTB1 (GTX46866) and PCNA (GTX100539) were purchased from GeneTex (San Antonio, TX, USA). The antibodies against PKN2 (35490) and c-Jun (13361) were purchased from Signalway Antibody (College Park, MD, USA). The antibodies against CyclinD1 (2978), CDK4 (12790), Bcl2 (3498), Bax (2772), Caspase-3 (9662), Caspase-9 (9508), p-ERK1/2 (9102), ERK1/2 (4370), AKT (4691), p-AKT (4060), p-cJun (3270), p-p38 MAPK(9216), p38 MAPK(8690) and GAPDH (2118) were purchased from Cell Signaling Technology (Danvers, MA, USA). The antibody against SNTB1 (PA5-55143), Fetal Bovine Serum (FBS), Trypsin-EDTA (0.25\%), Pierce TM BCA Protein Assay kit, and FxCycle PI/RNase Staining Solution was purchased from Thermo Fisher Scientific (Waltham, MA, USA). Annexin-V-AbFluor ${ }^{\text {TM }}$ 647 Apoptosis Detection kit and CCK8 kit were obtained from Abbkine (Wuhan, Hubei, China).

\section{Differential expressed genes analysis}

In prior work, we screened DEGs on 14 pairs of CRC primary lesions and surrounding non-cancerous tissues (GEO Submission: GSE113513) [11]. Among these DEGs, 8 of DEGs including SNTB1 were selected for further investigation due to the limited amount of published data regarding their involvement 
in carcinogenesis. In this study, mRNA expression of SNTB1in CRC and control tissues was analyzed thorough TCGA (https://cancergenome.nih.gov/) [12].

\section{Cell lines and culture}

Both human CRC cell lines HCT116 and RKO were obtained from the Cell Bank of the Chinese Academy of Sciences (Shanghai, China). HCT116 cells were cultured in M5'A medium (KeyGEN, Jiangsu, China) and RKO cells were cultured in MEM-alpha medium (Thermo Fisher Scientific) supplemented with $10 \%$ fetal bovine serum (FBS, Thermo Fisher Scientific), 1\% penicillin-streptomycin (Hyclone, Logan, UT, USA). All cells were cultured at $37^{\circ} \mathrm{C}$ in a humidified atmosphere with $5 \% \mathrm{CO}_{2}$. Short tandem repeat genotyping was performed for examination of mycoplasma contamination of cells using qPCR.

\section{Lentiviral transduction and high-content screening for cell growth}

High-content screening (HCS) was performed to assess the growth of CRC cells. Briefly, control shRNA lentivirus and shRNA lentivirus targeting 8 DEGs (the sequences of shRNAs are listed in Supplement Table S1) were constructed by Shanghai GeneChem (Shanghai, China). HCT116 cells were seeded in 12well plates for $16 \mathrm{~h}$ prior to lentivirus transduction and then transduced by adding the shRNA lentiviral particles (multiplicity of infection: 10) with GFP into the cell culture medium according to the protocol of the manufacturer. At the end of transduction, $2 \times 10^{3}$ cells in $100 \mu \mathrm{L}$ of complete medium were reseeded into 96-well plates. Cellomics Array Scan VTI HCS (Thermo Fisher Scientific) was used to monitor the cell growth over 5 days. Image analysis was performed using HCS Studio Cell Analysis Software (Thermo Fisher Scientific).

\section{Quantitative real-time PCR (qPCR) analysis}

The total RNA was isolated from cells using RNAiso Plus reagent (Takara, Beijing, China). Reverse transcription into complementary DNA (cDNA) was amplified according to the manufacturer's instructions using the PrimeScript RT reagent kit (Takara). Tissue cDNA array containing 79 primary CRC and 15 noncancerous colorectal tissues (Cat\#: cDNA-hcola095su01) was purchased from Shanghai Outdo Biotech Company (Shanghai, China) and the levels of mRNA encoding SNTB1 and GAPDH were detected using an ABI 7500 Fast Real-Time PCR System (Applied Biosystems) and the SYBR Premix Ex Tag (Takara). The conditions for qPCR were as follows: pre-denaturation $\left(95^{\circ} \mathrm{C}\right.$ for 10 minutes), denaturation $\left(95^{\circ} \mathrm{C}\right.$ for 15 seconds), annealing and extension $\left(60^{\circ} \mathrm{C}\right.$ for 60 seconds) for a total 40 cycles. GAPDH was used as an internal control. Primer sequences are shown in Supplementary Table S2. mRNA levels are presented as: $2 \Delta \Delta \mathrm{Ct}$ (with $\mathrm{Ct}$ being the cycle threshold), where $\Delta \mathrm{Ct}$ [Ct (target gene) - Ct (GAPDH)]. Clinicopathologic features of CRC patients represented in the cDNA array are summarized in Supplement Table S3.

\section{Tissue microarray array}

TMA containing 70 pairs of CRC and noncancerous colorectal tissues were obtained from Shanghai Outdo Biotech Company (Shanghai, China; Cat\#: HColA180Su15). IHC was conducted to detect the 
expression of SNTB1 in CRC samples using an antibody against SNTB1 (Rabbit monoclonal to SNTB1; dilution 1:100; Thermo Fisher Scientific), as described previously [13]. Scoring was carried out using a grading system based on staining intensity (no staining, 0; weak, 1; moderate, 2; strong, 3) and percentage of positive-staining cells ( $1-25 \%$ positive, $1 ; 26-50 \%, 2 ; 51-75 \%, 3 ; 76-100 \%, 4)$ [14]. The final score was calculated as intensity score $\times$ percentage score. Clinicopathological features of CRC patients are summarized in Supplement Table S4.

\section{Western-blot analysis}

Cells were harvested and lysed in RIPA lysis buffer (Thermo Fisher Scientific) containing $1 \mathrm{mM}$ phenylmethylsulfonyl fluoride (PMSF) and protease inhibitors. The Pierce BCA Protein Assay Kit (Thermo Fisher Scientific) was used to measure concentrations of total protein. Equal amount of total protein lysate was separated on $10 \%$ SDS-polyacrylamide gel and transferred to PVDF membranes (Millipore, Bedford, MA, USA). Next the membranes were blocked with $5 \%$ skim milk in TBST at room temperature for $2 \mathrm{~h}$ and incubated overnight at $4{ }^{\circ} \mathrm{C}$ with primary antibodies $(1: 1000)$, and followed by incubation with a horseradish peroxidase (HRP)-conjugated goat anti-rabbit secondary antibody (1:2000). Proteins were visualized using an ECL imager (Thermo Fisher Scientific, USA) and band intensities were quantified using ImageLab software. The expression of GAPDH was used as a control. Three independent experiments were performed for each assay.

\section{CCK-8 assay}

Transduced cells were re-seeded into 96 -well plates $\left(2,000\right.$ cells per well) and cultured in at $37^{\circ} \mathrm{C}$ and $5 \%$ $\mathrm{CO}_{2}$ for the indicated time points. Cell Counting Kit-8 reagent (10 ul; Abbkine, Wuhan, Hubei, China) was added to each well, plates were incubated for an additional $2 \mathrm{~h}$ at $37^{\circ} \mathrm{C}$, and the optical density (OD) was measured at a wavelength of $450 \mathrm{~nm}$. The cell viability was calculated based on the OD for each group.

\section{Colony formation assay}

Transduced cells were seeded into 12-well plates at a density of 500 cells per well and cultured at $37^{\circ} \mathrm{C}$ and $5 \% \mathrm{CO}_{2}$ for 10-14 days. Cells were fixed in $4 \%$ paraformaldehyde for $20 \mathrm{~min}$ and stained with $0.1 \%$ crystal violet (Solarbio, Beijing, China) for $20 \mathrm{~min}$ at room temperature. Colonies were manually counted. Each assay was performed in triplicate.

\section{Cell cycle and apoptosis analysis}

For the cell cycle assay, transduced cells were collected and fixed with $70 \%$ ethanol at $4{ }^{\circ} \mathrm{C}$ overnight. The fixed cells were centrifuged at $2000 \mathrm{rpm}$ for $3 \mathrm{~min}$ and washed, followed by incubation with a mixture of FxCycle PI/RNase Staining Solution (Thermo Fisher Scientific) for $30 \mathrm{~min}$ at room temperature. FACS (Fluorescence activated Cell Sorting; Becton Dickinson, CA, USA) was used to analyze cell cycle progression using ModfitLT version 3.0 (Verity Software House). For the apoptosis assay, transduced cells were washed twice with ice-cold PBS and incubated with Annexin-V-AbFlour ${ }^{\mathrm{TM}} 647$ Apoptosis Detection Kit solution (Abbkine, Wuhan, China). The apoptotic rate was analyzed using FACS. 
In vivo experiments

A xenograft nude mouse model was constructed to investigate the effects of SNTB1 knockdown on tumor growth. Male BALB/c nude mice (6-8 week old) were obtained from Shanghai Laboratory Animal Center at the Chinese Academy of Sciences and raised in a specific pathogen-free facility at Fujian University of Traditional Chinese Medicine (Fujian, China). All animal procedures were approved by the Committee of Fujian University of Traditional Chinese Medicine (Approval No: FJTCM IACUC 2019050). HCT116 cells or RKO cells were transduced with a lentivirus encoding anti-SNTB1 shRNA (sh-SNTB1) or control shRNA (sh-Ctrl). Cells $\left(1 \times 10^{6}\right.$ in $100 \mu \mathrm{L}$ PBS containing 50\% Matrigel) were injected subcutaneously into the flanks of nude mice $(n=6)$. Tumor volume was determined every other day using measurements obtained with a vernier caliper and the following formula: 1/2 (larger diameter×smaller diameter $^{2}$ ). At the end of the experiment, mice were anesthetized with isoflurane. An IVIS Spectrum liveanimal imaging system (PerkinElmer; Santa Clara, CA, USA) was used to capture tumor images. Mice were sacrificed for tissue collection for further use. Signal intensity was quantified as the number of photons within the region of interest per second.

\section{Immunohistochemistry}

Tissues were fixed with $4 \%$ paraformaldehyde at $4^{\circ} \mathrm{C}$ overnight, embedded with paraffin, cut into $5 \mu \mathrm{m}$ thick sections, and mounted onto slides. The slides were dehydrated by graded ethanol. Antigens were retrieved by using microwave heating for $20 \mathrm{~min}$ in sodium citrate-hydrochloric acid buffer. Tissue sections were incubated with an antibody against SNTB1 (1:100; Thermo fisher Scientific) or PCNA (1:800; Genetex). Background staining was assessed by omitting the primary antibody. The intensity of staining was evaluated using a scoring system described in detail in the Section "Tissue microarray array". The overall staining score was calculated by multiplying the intensity score and percentage score together.

\section{TUNEL assay}

Apoptotic cells in tissue sections were detected using terminal deoxynucleotidyl transferase dUTPnick end labeling (TUNEL) staining according to the manufacturer's instruction. The percentage of TUNELpositive cells and staining intensity were evaluated using a scoring system described in detail in the Section "Tissue microarray array".

\section{Isobaric tags for relative and absolute quantification (iTRAQ) analysis and protein identification.}

iTRAQ was used to identify differential expressed proteins (DEPs) $[15,16]$. SDS-PAGE electrophoresis was first carried out for protein quantification. The protein samples were cysteine-blocked and digested, protein labelling and mass spectrometry (MS) analysis were performed. Two-dimensional liquid chromatography-mass spectrometry (2D-LC-MSMS) analysis including reversed-phase chromatographic separation (Agilent Technologies, Santa Clara, CA, USA) and reversed-phase chromatography on a TripleTOF (AB SCIEX, Framingham, MA, USA) was conducted. Proteins were classified as differentially expressed if their expression differed at least 1.5-fold between the two conditions and if the difference 
was associated with $\mathrm{P}<0.05$. These proteins were identified using volcano plots and hierarchical clustering plots.

To enrich the biological groups and KEGG pathway, the identified proteins were submitted to Omicsbean (http://www.omicsbean.cn/) software. The significantly enriched gene ontology (GO) categories were reported using a right-sided hypergeometric test, which compares the background set of $\mathrm{GO}$ annotations in the whole genome of homo sapiens. The false discovery rate (FDR) was controlled by the Bonferroni step-down test to correct the p-value $[17,18]$.

To better understand the protein-protein interactions among the differentially expressed proteins of each group, we constructed protein-protein interaction (PPI) networks through Omicsbean. Proteins were then grouped based on their GO annotations with p-value < 0.05 [19].

\section{Statistics analysis}

Data were analyzed using SPSS 22.0 software. For survival analysis, SNTB1 mRNA expression in CRC tissues from cDNA array was classified into high or low expression groups based on mean. Kaplan-Meier survival curves were plotted for high- and low-expression groups and the correlation of SNTB1 mRNA expression with overall survival of CRC patients was analyzed using log-rank test. The correlation between SNTB1 mRNA expression and CRC patients' survival in dataset of COAD sourcing from TCGA was analyzed through Kaplan-Meier Plotter (http://kmplot.com/). Student's t-test or Mann-Whitney U was used for comparisons between two groups. One-way ANOVA or Kruskal-Walis $\mathrm{H}$ was applied to assess multiple group comparisons. All quantitative data are presented as the mean $\pm \mathrm{SD}$. $\mathrm{P}<0.05$ (two sided) was considered statistically significant. All experiments were repeated at least three times.

\section{Results}

\section{Identification of SNTB1 as a potential target in CRC}

In a previous experiment to identify potential oncogenes, we evaluated for differential expressed genes (DEGs) within a CRC microarray containing 14 pairs of $C R C$ tissue and its noncancerous surrounding tissue (GEO ID: GSE113513). Among the identified DEGs, we focused on 8 up-regulated genes (SNTB1, PLEKHG4, JPH1, CTPS1, LRRC6, LY6G6F, PLCB4 and LRP8; Fig. 1a) that had not been extensively studied in CRC. The potential involvement of these genes in CRC were further explored through high-content screening (HCS) using lentivirus delivered shRNAs into HCT116 cells. Seven of the 8 constructs inhibited cell growth (Fig. 1b, c). Considering the unexplored role of SNTB1 in oncogenesis [9, 11, 20, 21], we focused on SNTB1 in these studies.

\section{SNTB1 is highly expressed in CRC tissues and is associated with poor prognosis}


QPCR analysis on a cDNA array of 79 primary CRC and 15 noncancerous colorectal tissues indicated that SNTB1 mRNA expression is up-regulated in CRC tissues (Fig. 2a). Similar results were obtained with online data mining using the GEO (GEO ID: GSE113513) and TCGA (Fig. 2b,c) datasets. In a consistent fashion, immunohistochemical (IHC) evaluation of a tissue microarray (TMA) containing 70 pairs of primary CRC lesions and adjacent noncancerous tissues (Fig. 2d, e) confirmed the up-regulation of SNTB1 protein expression in CRC tissues. Correlation analysis of the CRC patients represented in the utilized cNDA array, showed that higher SNTB1 expression (as determined with QPCR-based cDNA array) correlated with shorter overall survival rate (Fig. 2f). There was no correlation between SNTB1 expression and clinicopathological characteristics of CRC patients (Supplementary Table 5). Online data mining using the dataset of COAD from TCGA also revealed an association between higher SNTB1 expression and shorter overall survival of CRC patients (Fig. 2g).

\section{SNTB1 knockdown inhibits cell proliferation and induces cell apoptosis of CRC cells in vitro}

To explore the biological function of SNTB1 on CRC cell growth, three different shRNAs specific for SNTB1 were encoded within lentiviruses and used for cell transduction. QPCR and Western-blot analysis revealed significant down-regulation of SNTB1 expression of both mRNA (Supplementary Fig. 1a) and protein (Supplementary Fig. 1b, c) levels in HCT116 cells with all three shRNAs. As each of the shRNAs had similar effects on SNTB1 expression and cell growth, subsequent experiments were performed with a single construct referred to as "sh-SNTB1-1".

QPCR and Western-blot analysis confirmed significant down-regulation of SNTB1 mRNA and protein levels in both HCT116 and RKO cells after transduced with sh-SNTB1 (Fig. 3a-c). More importantly, SNTB1 knockdown profoundly reduced the viability and colony formation of HCT116 and RKO cells (Fig. 3d, e). Moreover, SNTB1 knockdown markedly increased percentage of cells in the G0/G1 phase, while decreased cell percentage in the S phase in HCT116and RKO cells (Fig. 4a), as well as downregulated the expression of G0/G1-related proteins CDK4 and CyclinD1 in HCT116 cells (Fig. 4b). Flow cytometry with annexin $\mathrm{V}$ staining and western-blot analysis demonstrated that SNTB1 knockdown remarkably increased the percentage of apoptotic cells (Fig. 4c) and the ratio of Bax/Bcl-2 expression (Fig. 4d).

\section{SNTB1 knockdown suppresses CRC tumor growth in vivo}

We further determined the effects of SNTB1 knockdown on tumor growth in vivo. By evaluating tumor volume (Fig. 5a), intratumoral green fluorescent protein (GFP) fluorescence (Fig. 5b, c), tumor size (Fig. 5d), and tumor weight (Fig. 5e), we found that SNTB1 knockdown significantly suppressed tumor growth of both HCT116 and RKO cells in vivo. IHC analysis and TUNEL assay showed that SNTB1 knockdown significantly decreased the expressions of SNTB1 (Fig. 6a) and proliferating cell nuclear antigen (PCNA) (Fig. 6b), and increased cell apoptosis in the HCT116 xenograft (Fig. 6c). 


\section{SNTB1 knockdown up-regulates PKN2 expression and inhibit the activation of ERK and AKT signaling pathways}

To further elucidate the underlying mechanism of SNTB1 knockdown on tumor growth suppression in CRC, the isobaric tag for relative and absolute quantitation (iTRAQ) methodology was applied to identify differentially expressed proteins (DEPs) in HCT116 cells after SNTB1 knockdown. As shown in Fig. 7, a total of 265 DEPs were identified, including 210up-regulatedand 55down-regulated proteins (fold change $\geq 1.5, P<0.05$ ). Hierarchical clustering (Fig. 7a) and volcano (Fig. 7b) plots were used to identify DEPs. Analysis of Kyoto Encyclopedia of Genes and Genomes (KEGG) pathways showed these proteins to be enriched in multiple signaling pathways (Fig. 7c). A PPI network was constructed (Fig. 7d) and the top 10 hub genes were identified on the basis of their degree of connectivity in the PPI network: PKN2, TMX3, DYRK1A, IRAK1, SYNE2, HELZ, GSK3A, MY05B, and NVL (Supplement Table 6).

As a top hub protein, PKN2 expression was significantly up-regulated in HCT116 cells after SNTB1 knockdown. Therefore, we further explored the regulatory effects of SNTB1 knockdown on PKN2 expression and its downstream targets, including ERK and AKT pathways. Consistently, SNTB1 knockdown significantly increased the protein pression of PKN2 (Fig. 7e) and inhibited the phosphorylation of ERK (Fig. 7f) and AKT (Fig. 7g). However, SNTB1 knockdown had no significant effects on the phosphorylation of c-jun and p38 MAPK in HCT116 cells (Supplement Fig. 2).

\section{Discussion}

$\mathrm{CRC}$ is one of the most lethal cancers worldwide [1]. Although unhealthy dietary habits, environmental changes, and genetic aberrancies are contributors to the morbidity and mortality of this disease [22], the precise molecular mechanisms of CRC initiation and progression remain elusive. Therefore, the investigation into the molecular determines of CRC has the potential to lead to novel therapeutic targets, and diagnostic and prognostic biomarkers. Herein, we for the first time describe the potential role of SNTB1 as a oncogene in CRC tissues.

As a modular adapter protein, SNTB1 binds and localizes various transmembrane and intracellular signaling molecules to the membrane [23]. Our current study identified the up-regulation of SNTB1 in CRC tissues in both mRNA and protein levels, suggesting that up-regulation of SNTB1 might be a common event during the development of CRC. Moreover, survival analysis based on CDNA array of CRC samples and online public databases indicate that there is a correlation between higher SNTB1 expression and shorter survival of CRC patients. This finding highlights the potential of SNTB1 as a biomarker for disease prognosis.

Previous studies indicate that SNTB1 is involved in the initiation of autophagy in pancreatic cancer cells and has a protective effect on acute pancreatitis [7]. In addition, it has also been reported that SNTB1 is closely related to the occurrence of oral cancer and is considered to be a susceptibility locus for severe myopia $[9,10]$. However, the functional roles of SNTB1 in CRC are still unknown. In order to further 
understand the function of SNTB1, we conducted a series of experiments and found that SNTB1 knockdown significantly suppressed CRC cell growth in vivo and in vitro by inhibiting cell proliferation and inducing cell cycle progression and cell apoptosis. These findings suggesting a potential oncogenic activity of endogenous SNTB1 in CRC. However, the effect of SNTB1 overexpression in CRC cell growth should be addressed in future study.

The involvement of SNTB1 in cell proliferation and apoptosis is unknown, we therefore conducted iTRAQ analysis to identify differentially expressed proteins in CRC cells after SNTB1 knockdown. iTRAQ analysis identified a total of 265 differentially expressed proteins (including 210 upregulated and 55 downregulated proteins) after knockdown of SNTB1. To identify the downstream targets of SNTB1, a PPI network was constructed. PPI analysis identified PKN2 as a hub protein in the PPI network, which was down-regulated in CRC cells after SNTB1 knockdown. Previous studies indicate that PKN2 is involved in cell proliferation by regulating ERK/MAPK or AKT signaling pathways $[24,25]$ and inhibit M2 phenotype polarization of tumor-associated macrophages in CRC cells [26]. Since abnormal activation of MAPK and AKT pathways contribute to multiple cellular processes, including cell survival, cell differentiation, apoptosis, invasion, and inflammation [27-30], we therefore assessed the regulatory effects of SNTB1 on those multiple signaling pathways. Interestingly, SNTB1 knockdown significantly decreased the levels of both p-ERK and p-AKT expression, without effect on p38 MAPK and p-JNK. However, the exactly regulatory role of SNTB1 on PKN2 expression and EKR, AKT pathways activation should be addressed in future studies.

\section{Conclusion}

In summary, our current study indicates that SNTB1 expression is upregulated in CRC tissues and correlates with CRC patient survival. In addition, we demonstrate SNTB1 has an essential role in CRC proliferation and apoptosis, which is mediated, in part, through the reduction of PKN2 expression and activation of EKR and AKT pathways. Also, these studies highlight the potential of SNTB1 as a therapeutic target and biomarker for CRC.

\section{Abbreviations}

CRC

Colorectal cancer; SNTB1:Syntrophin beta 1; iTRAQ:isobaric tags for relative and absolute quantification; PKN2:protein kinase N2; PPI:protein-protein interaction; TUNEL:terminal deoxynucleotidyl transferase dUTPnick end labeling; PCNA:proliferating cell nuclear antigen.

\section{Declarations}

\section{Ethics approval and consent to participate}

Not applicable. 


\section{Consent for publication}

Not applicable.

\section{Competing interests}

The authors have declared that no competing interest exists.

\section{Funding}

This study was supported by the National Natural Science Foundation of China (81803882), and the Natural Science Foundation of Fujian Province (2020J06026).

\section{Authors' contributions}

$L L, Y C, X L, J P$ and $A S$ conceived and designed the study. $L L, X L, L C$ and $J L$ performed the animal experiments. YC, QX, YH and MY performed the cell experiments. LL, MW and HL performed the iTRAQ assay and analysis. LL, XL and TS produced the initial draft of the manuscript. YC, TS, JP and AS revised the manuscript. All authors read and approved the final manuscript.

\section{Acknowledgements}

Not applicable.

\section{Availability of data and materials}

The datasets generated and analyzed during the current study are available from the corresponding author on reasonable request.

\section{Author details}

${ }^{1}$ Academy of Integrative Medicine, Fujian University of Traditional Chinese Medicine, 1 Qiuyang Road, Minhou Shangjie, Fuzhou, Fujian 350122, China. ${ }^{2}$ Fujian Key Laboratory of Integrative Medicine on Geriatrics, Fujian University of Traditional Chinese Medicine, 1 Qiuyang Road, Minhou Shangjie, Fuzhou, Fujian 350122, China. ${ }^{3}$ Department of Pediatrics, Case Western Reserve University School of Medicine, Rainbow Babies and Children's Hospital, Cleveland, OH 44106, USA. ${ }^{4}$ Department of Physical Education, Fujian University of Traditional Chinese Medicine, Fuzhou, Fujian, 350122, China.

\section{References}


1. Sung H, Ferlay J, Siegel RL, Laversanne M, Soerjomataram I, Jemal A, et al. Global cancer statistics 2020: GLOBOCAN estimates of incidence and mortality worldwide for 36 cancers in 185 countries. CA Cancer J Clin. 2021;4. doi:10.3322/caac.21660.

2. Fatemi SR, Pourhoseingholi MA, Asadi F, Vahedi M, Pasha S, Alizadeh L, et al. Recurrence and FiveYear Survival in Colorectal Cancer Patients After Surgery. Iran J Cancer Prev. 2015;8(4):e3439.

3. Kassahun WT. Unresolved issues and controversies surrounding the management of colorectal cancer liver metastasis. World J Surg Oncol. 2015;13:61.

4. Marin JJ, Sanchez de Medina F, Castaño B, Bujanda L, Romero MR, Martinez-Augustin O, et al. Chemoprevention, chemotherapy, and chemoresistancein colorectal cancer. Drug Metab Rev. 2012;44(2):148-72.

5. Alessi A, Bragg AD, Percival JM, Yoo J, Albrecht DE, Froehner SC, et al. gamma-Syntrophin scaffolding is spatially and functionally distinct from that of the alpha/beta syntrophins. Exp Cell Res. 2006;312(16):3084-95.

6. Yoshizawa K, Inaba K, Mannen H, Kikuchi T, Mizutani M, Tsuji S. Analyses of beta-1 syntrophin, syndecan 2 and gem GTPase as candidates for chicken muscular dystrophy. Exp Anim. 2003;52:391-6.

7. Ye R, Onodera T, Blanchard PG, Kusminski CM, Esser V, Brekken RA, et al. $\beta 1$ Syntrophin Supports Autophagy Initiation and Protects against Cerulein-Induced Acute Pancreatitis. Am J Pathol. 2019;189(4):813-25.

8. Albrecht DE, Froehner SC. Syntrophins and dystrobrevins: defining the dystrophin scaffold at synapses. Neurosignals. 2002;11(3):123-9.

9. Khor CC, Miyake M, Chen LJ, Shi Y, Barathi VA, Qiao F, et al. Genome-wide association study identifies ZFHX1B as a susceptibility locus for severe myopia. Hum Mol Genet. 2013;22(25):5288-94.

10. Yete S, Pradhan S, Saranath D. Single nucleotide polymorphisms in an Indian cohort and association of CNTN4, MMP2 and SNTB1 variants with oral cancer. Cancer Genet. 2017;214:16-25.

11. Shen A, Chen Y, Liu L, Huang Y, Chen H, Qi F, et al. EBF1-Mediated Upregulation of Ribosome Assembly Factor PNO1 Contributes to Cancer Progression by Negatively Regulating the p53 Signaling Pathway. Cancer Res. 2019;79(9):2257-70.

12. Blum A, Wang P, Zenklusen JC. SnapShot: TCGA-Analyzed Tumors. Cell. 2018;5:173:530.

13. Wang Z, Fan M, Candas D, Zhang TQ, Qin L, Eldridge A, et al. Cyclin B1/Cdk1 coordinates mitochondrial respiration for cell-cycle G2/M progression. Dev Cell. 2014;29(2):217-32.

14. Jang SH, Kim AR, Park NH, Park JW, Han. IS.DRG2 Regulates G2/M progressionvia the Cyclin B1Cdk1 complex. Mol Cells. 2016;39:699-704.

15. Calderón-González KG, Valero Rustarazo ML, Labra-Barrios ML, Bazán-Méndez Cl, Tavera-Tapia A, Herrera-Aguirre ME, et al. Determination of the protein expression profiles of breast cancer cell lines by quantitative proteomics using iTRAQ labelling and tandem mass spectrometry. J Proteomics. $2015 ; 124: 50-78$. 
16. Ma Y, Xiao T, Xu Q, Shao X, Wang H. iTRAQ-based quantitative analysis of cancer-derived secretory proteome reveals TPM2 as a potential diagnostic biomarker of colorectal cancer. Frontiers in Medicine. 2016;10:278-85.

17. Li F, Zhao D, Yang S, Wang J, Liu Q, Jin X, et al. ITRAQ-Based Proteomics Analysis of Triptolide On Human A549 Lung Adenocarcinoma Cells. Cell Physiol Biochem. 2018;45(3):917-34.

18. Cai Y, Zeng M, Chen YZ. The pharmacological mechanism of Huashi Baidu Formula for the treatment of COVID-19 by combined network pharmacology and molecular docking. Ann Palliat Med. 2021;10(4):3864-95.

19. Zhou DD, Li HL, Liu W, Zhang LP, Zheng Q, Bai J, et al. miR-193a-3p Promotes the Invasion, Migration, and Mesenchymal Transition in Glioma through Regulating BTRC. Biomed Res Int. 2021;2021:8928509.

20. Galvan A, Frullanti E, Anderlini M, Manenti G, Noci S, Dugo M, et al. Gene expression signature of noninvolved lung tissue associated with survival in lung adenocarcinoma patients. Carcinogenesis. 2013;34(12):2767-73.

21. Lee Y, Park S, Lee SH, Lee H. Characterization of genetic aberrations in a single case of metastatic thymic adenocarcinoma. BMC Cancer. 2017;17(1):330.

22. Park Y, Park SJ, Cheon JH, Kim WH, Kim TI. Association of Family History With Cancer Recurrence, Survival, and the Incidence of Colorectal Adenoma in Patients With Colorectal Cancer. J Cancer Prev. 2019;24:1-10.

23. Albrecht DE, Froehner SC. Syntrophins and dystrobrevins: defining the dystrophin scaffold at synapses. Neurosignals. 2002;11(3):123-9.

24. Cheng $Y$, Zhu Y, Xu J, Yang M, Chen P, Xu W, et al. PKN2 in colon cancer cells inhibits M2 phenotype polarization of tumor-associated macrophages via regulating DUSP6-Erk1/2 pathway. Mol Cancer. 2018;17(1):13.

25. Lee SJ, Hwang J, Jeong HJ, Yoo M, Go GY, Lee JR, et al. PKN2 and Cdo interact to activate AKT and promote myoblast differentiation. Cell Death Dis. 2016;7(10):e2431.

26. Lin X, Han L, Gu C, Lai Y, Lai Q, Li Q, et al. MiR-452-5p promotes colorectal cancer progression by regulating an ERK/MAPK positive feedback loop. Aging. 2021;13(5):7608-26.

27. Kim EK, Choi EJ. Pathological roles of MAPK signaling pathways in human diseases. Biochim Biophys Acta. 2010;1802:396-405.

28. Samatar AA, Poulikakos PI. Targeting RAS-ERK signalling in cancer: promises and challenges. Nat Rev Drug Discov. 2014;13:928-42.

29. Cargnello M, Roux PP. Activation and function of the MAPKs and theirsubstrates, the MAPK-activated protein kinases. Microbiol Mol Biol Rev. 2011;75:50-83.

30. Weng YR, Kong X, Yu YN, Wang YC, Hong J, Zhao SL, et al. The role of ERK2 in colorectal carcinogenesis is partly regulated by TRAPPC4. Mol Carcinog. 2014;53(Suppl 1):E72-84. 


\section{Figures}

a

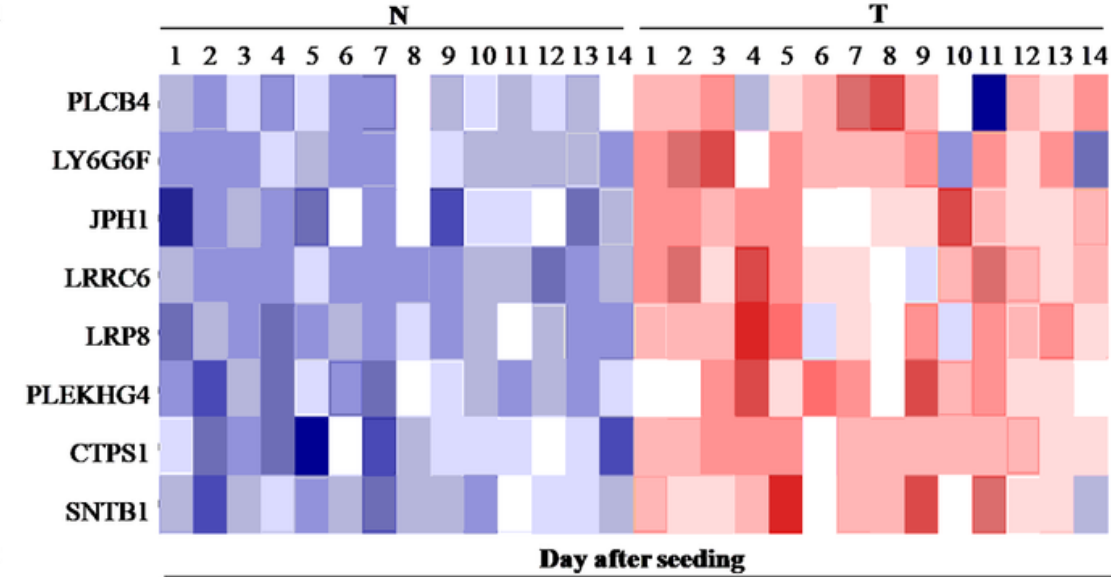

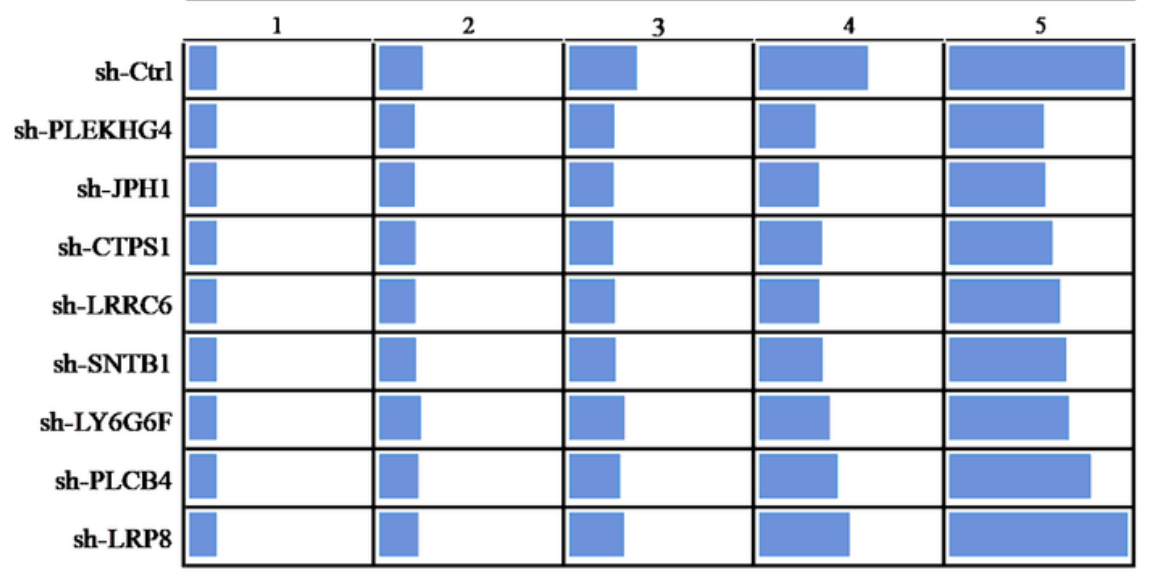

b

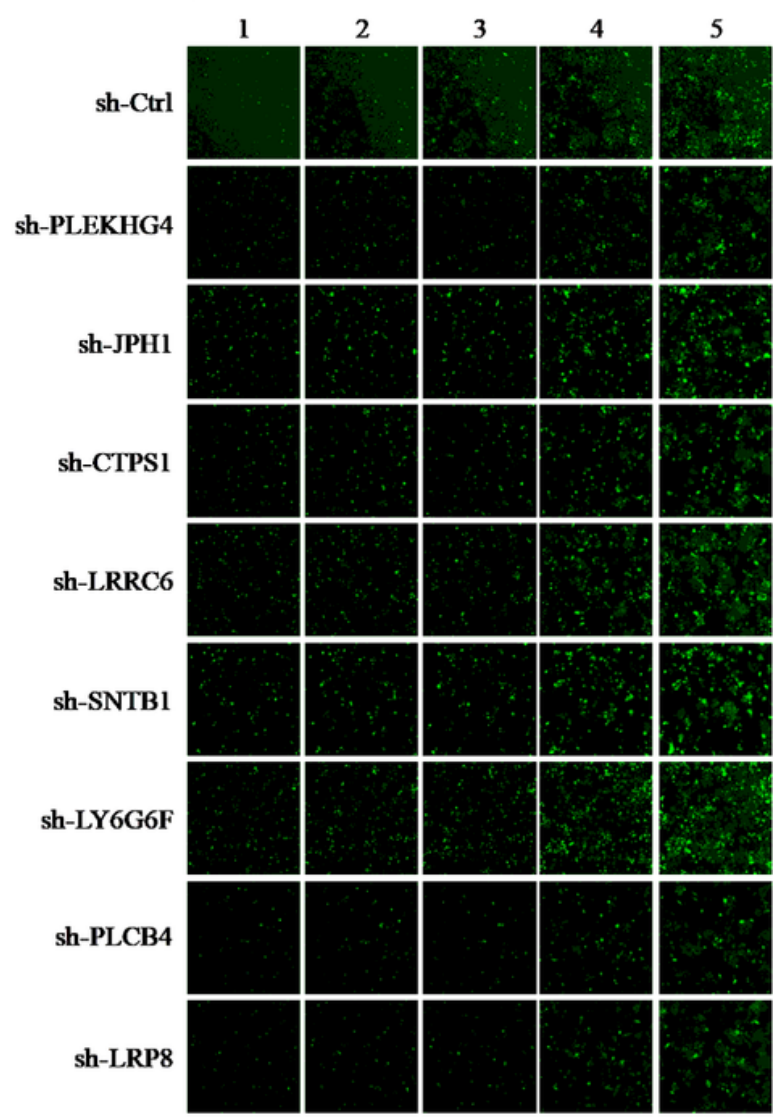

\section{Figure 1}

High-content shRNA screening identified SNTB1 as a potential oncogene in CRC. (a) Microarray analysis was performed to identify differentially expressed genes (DEGs) between 14 pairs of colorectal cancer $(\mathrm{CRC})$ tissues $(\mathrm{T})$ and adjacent normal tissues $(\mathrm{N})$. Heatmap of the 8 selected DEGs mRNA expression in CRC primary lesions $(T)$ and non-cancerous tissues $(N)$ in microarray analysis. HCT116 cells were transduced with lentivirus encoding shRNAs against these 8 DEGs (SNTB1, PLEKHG4, JPH1, CTPS1, LRRC6, LY6G6F, PLCB4 and LRP8), and cell growth was measured using the Cellomics ArrayScan VTI HCS. (b) Heatmap showing the growth of HCT116 cells. Data were normalized to cell number on day 1 and represented as fold change. (c)The representative images were taken at a magnification of 200x. 
$\mathbf{a}$
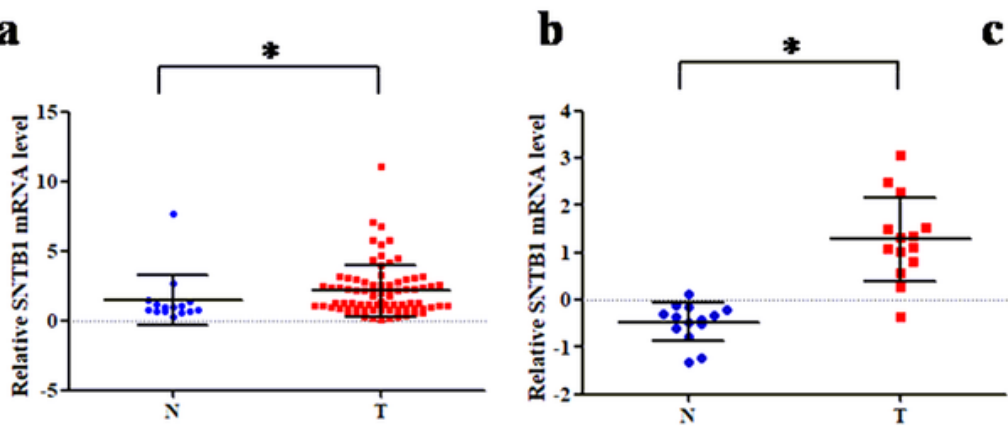

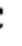

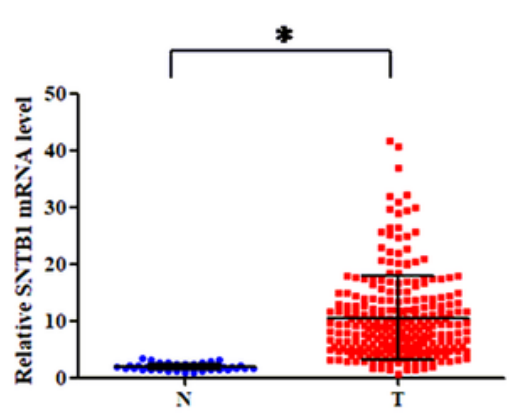

d

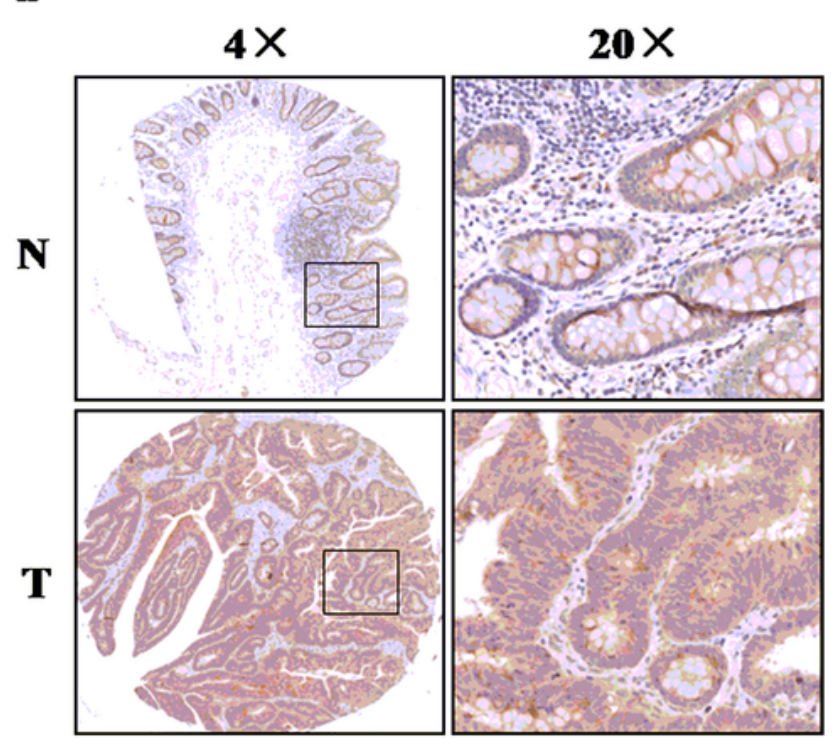

e

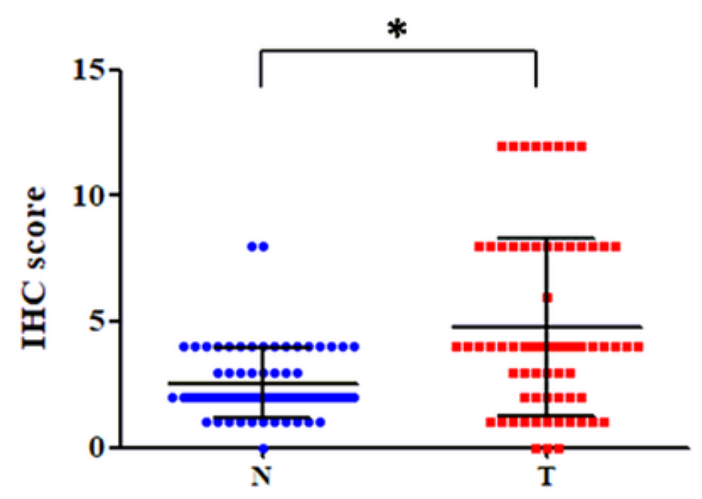

f

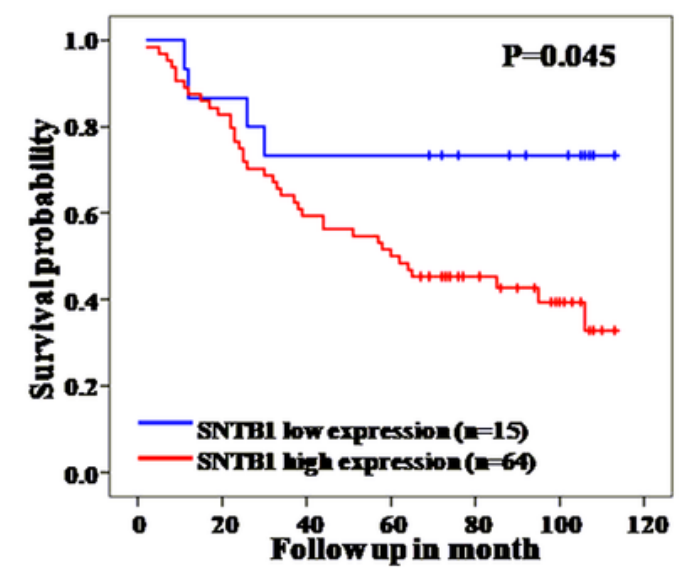

g

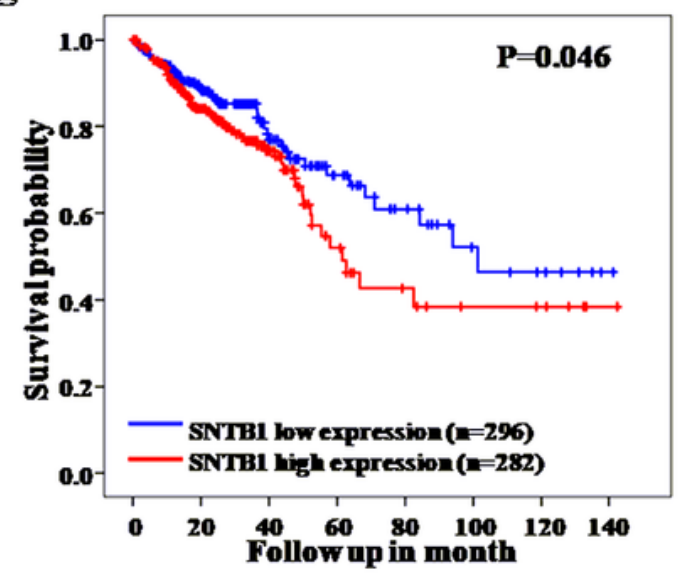

\section{Figure 2}

Levels of SNTB1 mRNA and protein in colorectal cancer tissues. (a) SNTB1 mRNA expression in CRC tissues ( $T: n=79)$ and non-cancerous colorectal tissues $(N: n=15)$ on the CRC cDNA chip was analyzed by qPCR. GAPDH was used as an internal control. P < 0.05 vs. N. (b and c) SNTB1 mRNA expression in CRC tissues (T) and non-cancerous colorectal tissues (N) from GEO (b) and TCGA (c) were analyzed. (d and e) SNTB1 protein expression in CRC tissues $(\mathrm{T})$ and non-cancerous colorectal tissues $(\mathrm{N})$ was determined by 
immunohistochemistry of a tissue microarray. Representative images were taken at magnification of $40 x$ or 200x (right panel); the IHC score was calculated as intensity score $\times$ percentage score (left panel, see Methods). $\mathrm{P}<0.05$ vs. N. (f and g) Kaplan-Meier plots of survival of CRC patients, stratified by SNTB1 mRNA expression based on CDNA array (f) and TCGA database (g). Survival was analyzed with log-rank test.

a

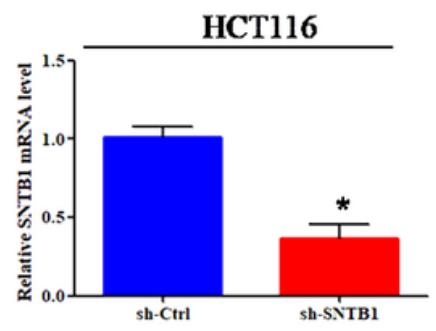

b

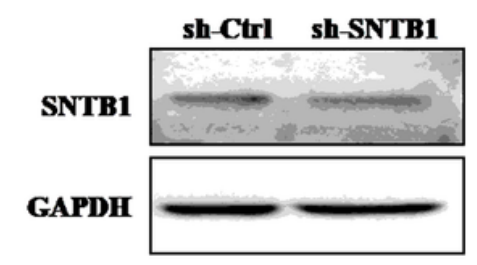

c

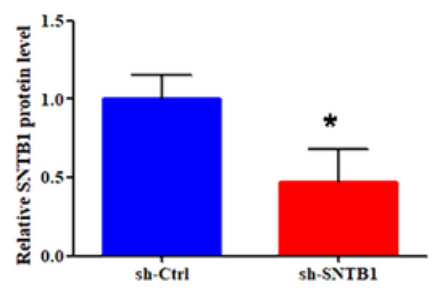

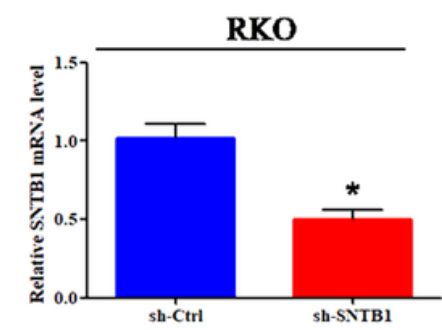
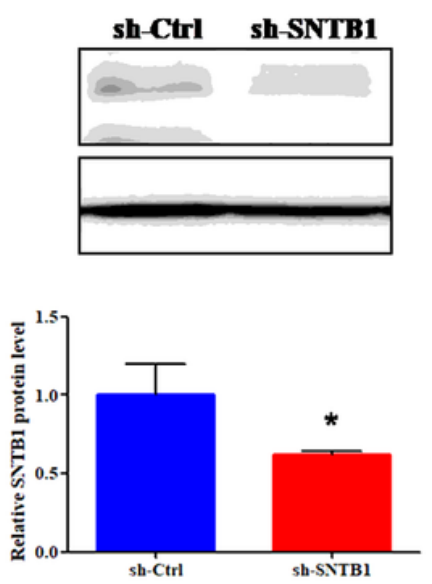

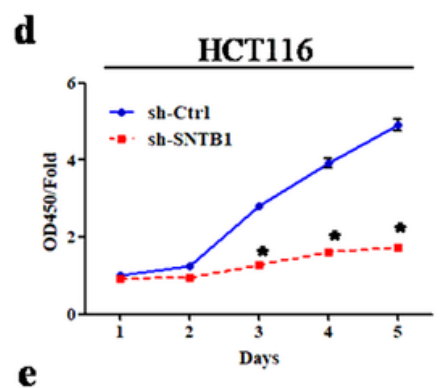

$\mathbf{e}$
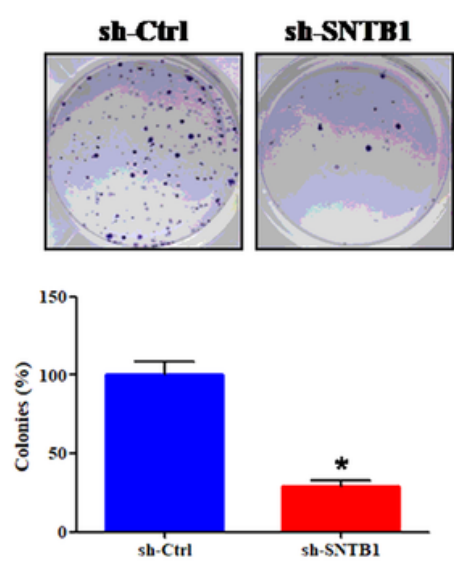
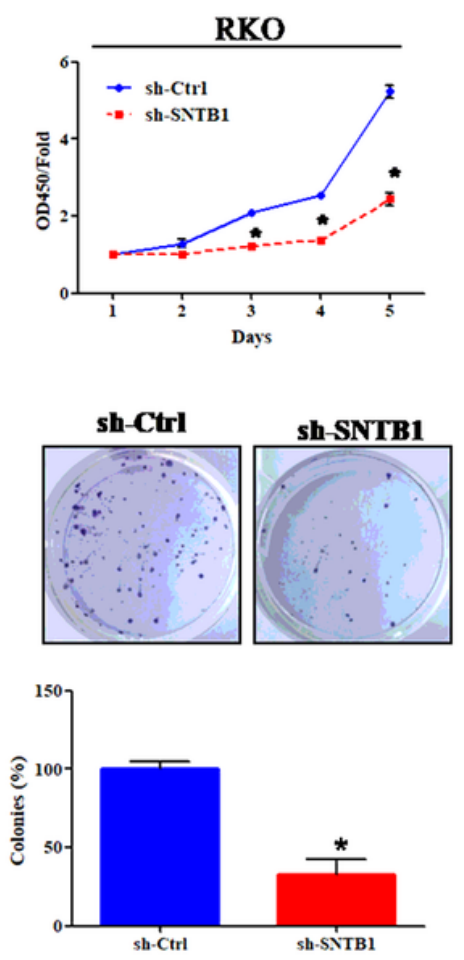

Figure 3

SNTB1 knockdown inhibits colorectal cancer (CRC) cell growth. HCT116 and RKO cells were transduced with lentivirus encoding either anti-SNTB1 small hairpin RNA (sh-SNTB1) or control shRNA (sh-Ctrl). (a)The mRNA levels of SNTB1 were determined by GPCR, GAPDH was used as an internal control. *P< 0.05 vs. sh-Ctrl lentivirus. ( $b$ and $c$ ) The protein levels of SNTB1 were determined by Western-blot analysis. The representative images of SNTB1 and GAPDH were showed (b) and were quantitated using ImageLab software (c). GAPDH was used as an internal control and normalized to GAPDH. *P $<0.05$ vs. sh-Ctrl. (d) The cell viability of CRC cells was determined by the CCK-8 assay. Data were normalized to the viability on Day 1 and are represented as fold change. ${ }^{*} P<0.05$ vs. sh-Ctrl. (e) The colony formation assay was performed to determine cell survival. The images were taken and the colonies formation were calculated, and normalized to the survival of control cells. ${ }^{*} \mathrm{P}<0.05$ vs. sh-Ctrl. 
a
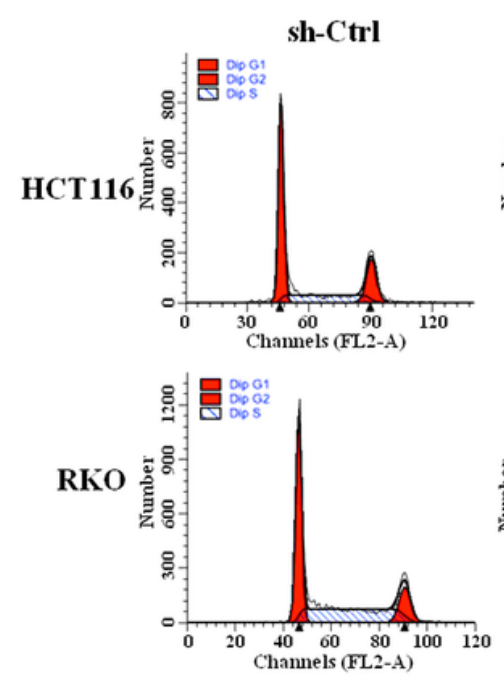

c
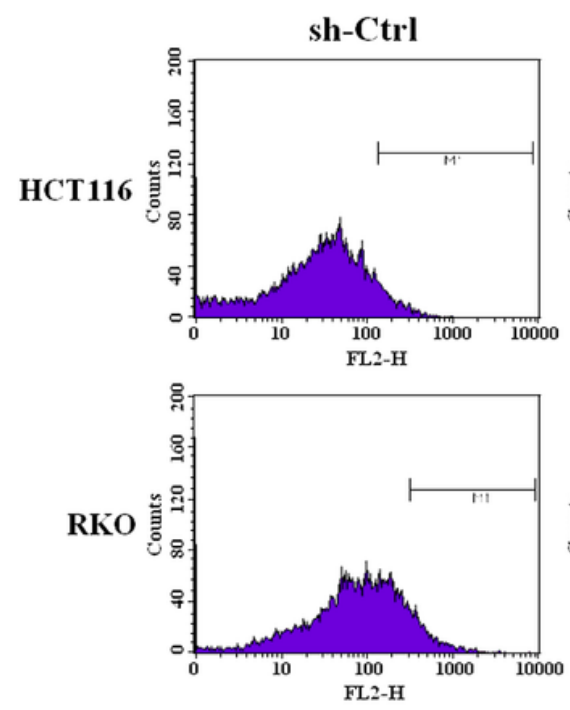

sh-SNTB1
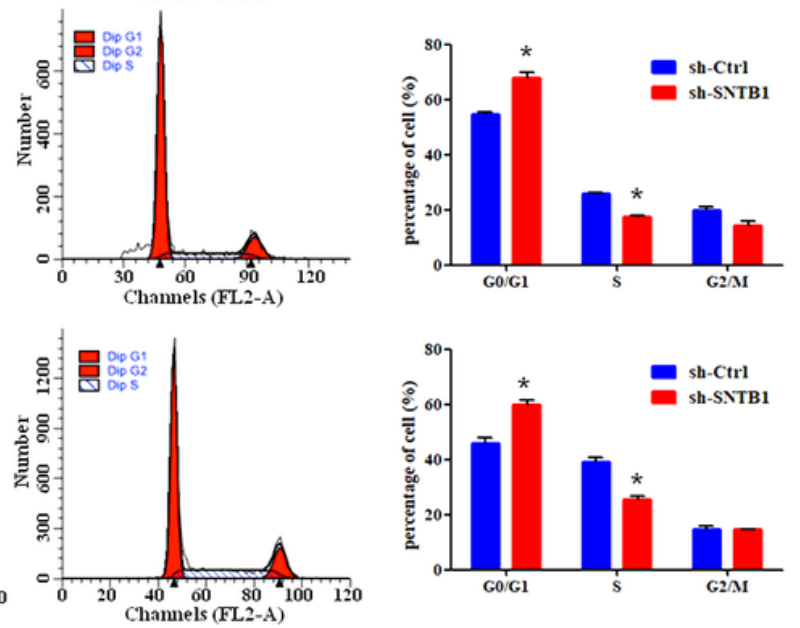

b
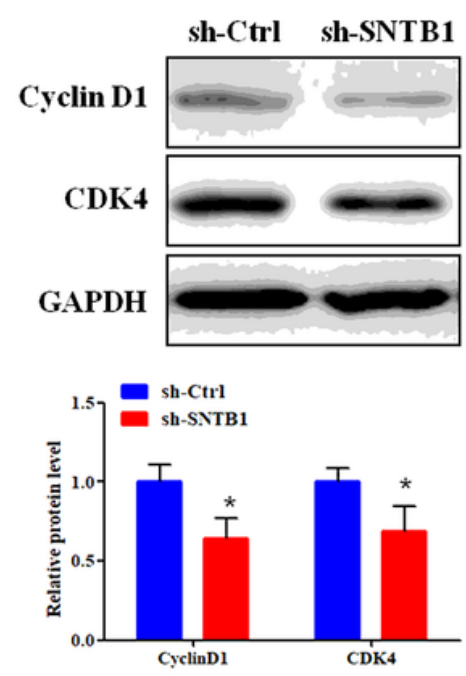

d
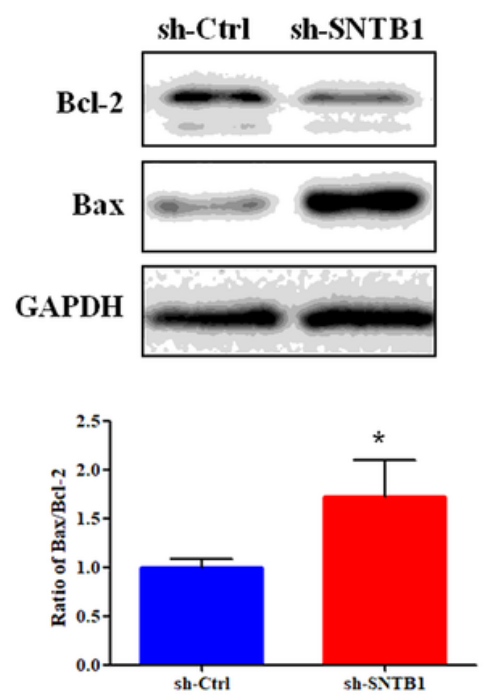

Figure 4

SNTB1 knockdown inhibits cell proliferation and induces cell apoptosis in colorectal cancer (CRC) cells. HCT116 and RKO cells were transduced with lentivirus encoding either anti-SNTB1 small hairpin RNA (shSNTB1) or control shRNA (sh-Ctrl). (a)The cell cycle progression in HCT116 (upper panel) and RKO (lower panel) cells was determined by PI staining and FACS analysis. Representative plots (left and middle panels) and percentage of cells (right panel) at different stages (G0/G1, G2/M and S phases) were presented. *P $<0.05$ vs. sh-Ctrl. (b) The protein levels of CDK4 and CyclinD1 in HCT116 cells after SNTB1 knockdown were determined by Western-blot analysis. The representative images were showed in upper panel and their expression were quantitated using ImageLab software (lower panel). GAPDH was used as an internal control. *P < 0.05 vs. sh-Ctrl. (c) The cell apoptosis of HCT116 (upper panel) and RKO (lower panel) cells was determined by Annexin $\mathrm{V}$ staining and FACS analysis. Representative plots (left and middle panels) and percentage of apoptotic cells (right panel) were presented. *P $<0.05$ vs. sh-Ctrl. (d) The protein levels of Bax and Bcl-2 in HCT116 cells after SNTB1 knockdown were determined by Westernblot analysis. The representative images were showed in upper panel and their expression were 
quantitated using ImageLab software (lower panel). GAPDH was used as an internal control. *P $<0.05$ vs. sh-Ctrl. All experiments were performed in triplicate.

$\mathbf{a}$

HCT16

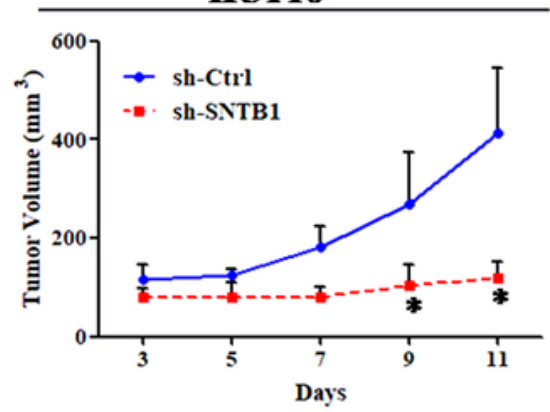

b

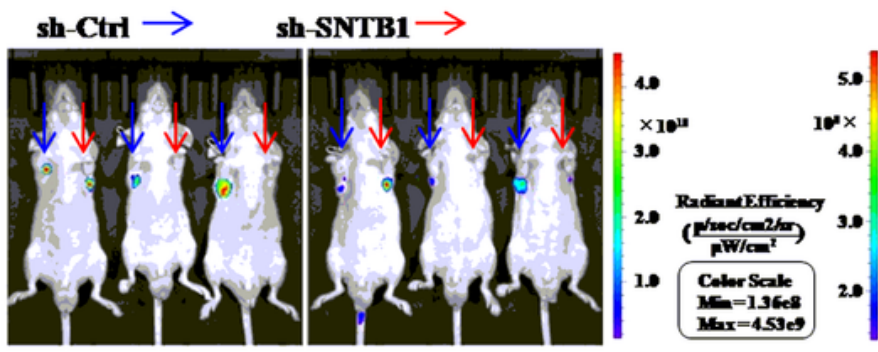

c

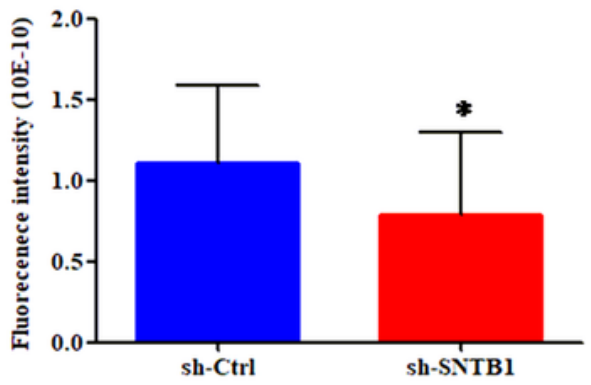

d

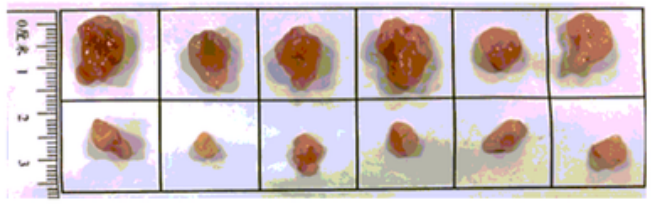

$\mathbf{e}$

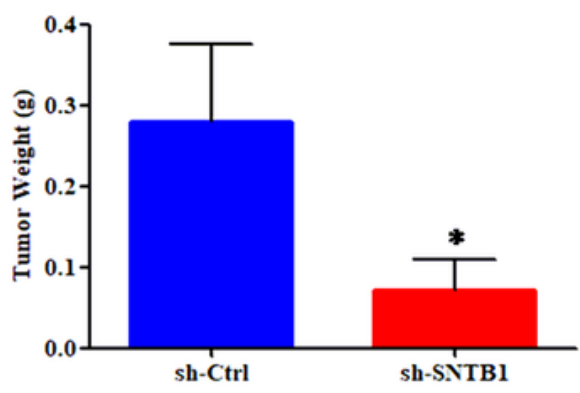

RKO

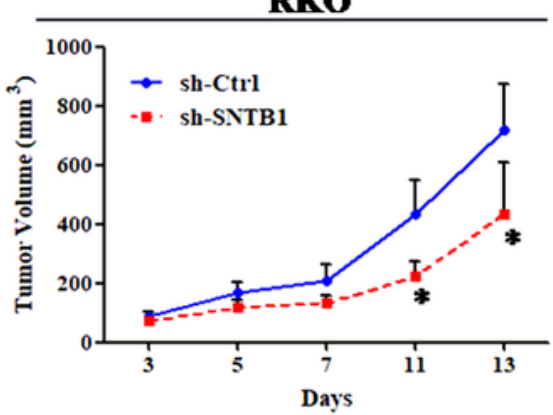

sh-Ctrl $\longrightarrow \quad$ sh-SNTB1 $\longrightarrow$
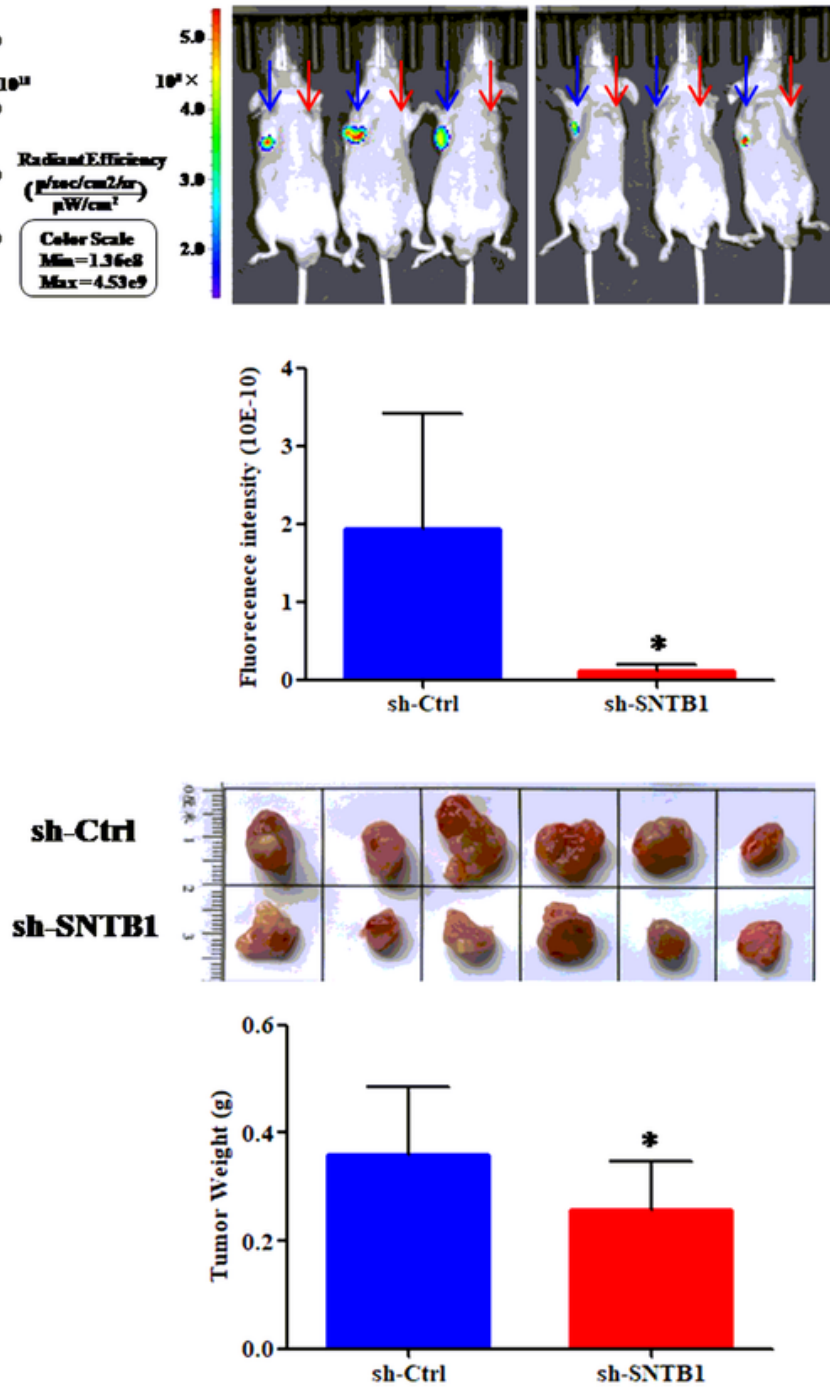

Figure 5

SNTB1 knockdown inhibits colorectal cancer (CRC) cell growth in vivo. A xenograft nude mouse model was established to investigate the tumor growth of HCT116 (left panel) and RKO (right panel) cells in vivo. A total of $1.0 \times 106$ transduced cells were injected subcutaneously into the armpits (sh-Ctrl: left 
armpit; sh-SNTB1: right armpit) of BALB/c nude mice; these cells had been transduced with lentivirus encoding sh-Ctrl (left armpit) or sh-SNTB1 (right armpit). The tumor volumes (a), fluorescence of GFP (b and $\mathrm{c}$ ), images of tumor and tumor weights ( $\mathrm{d}$ and e) were determined and recorded. ${ }^{*} \mathrm{P}<0.05 \mathrm{vs}$. sh-Ctrl.

$\mathbf{a}$

\section{SNTB1}
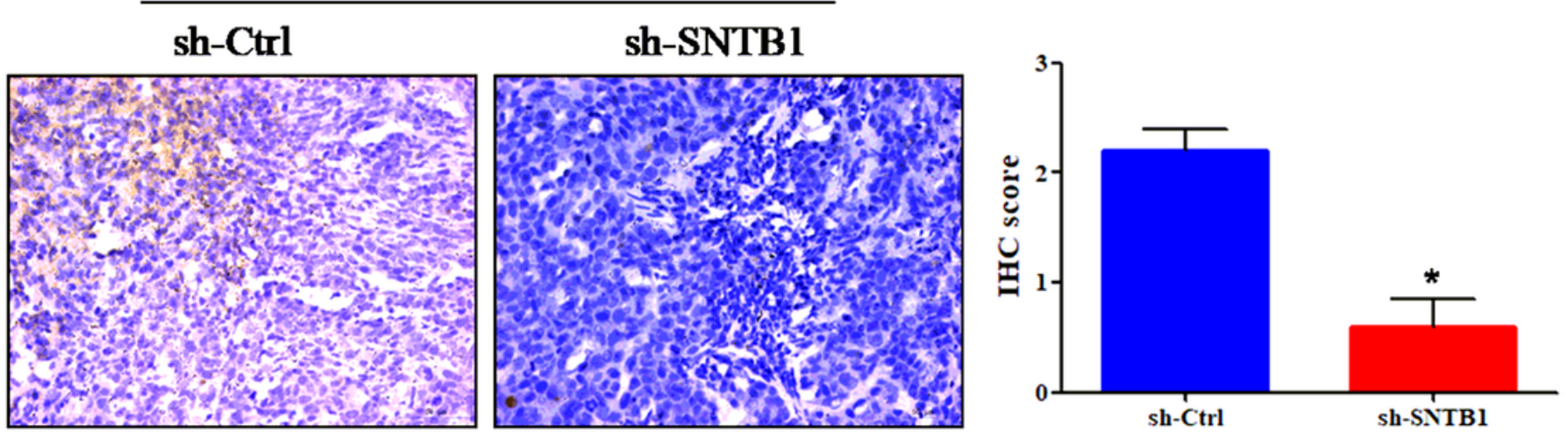

b

PCNA
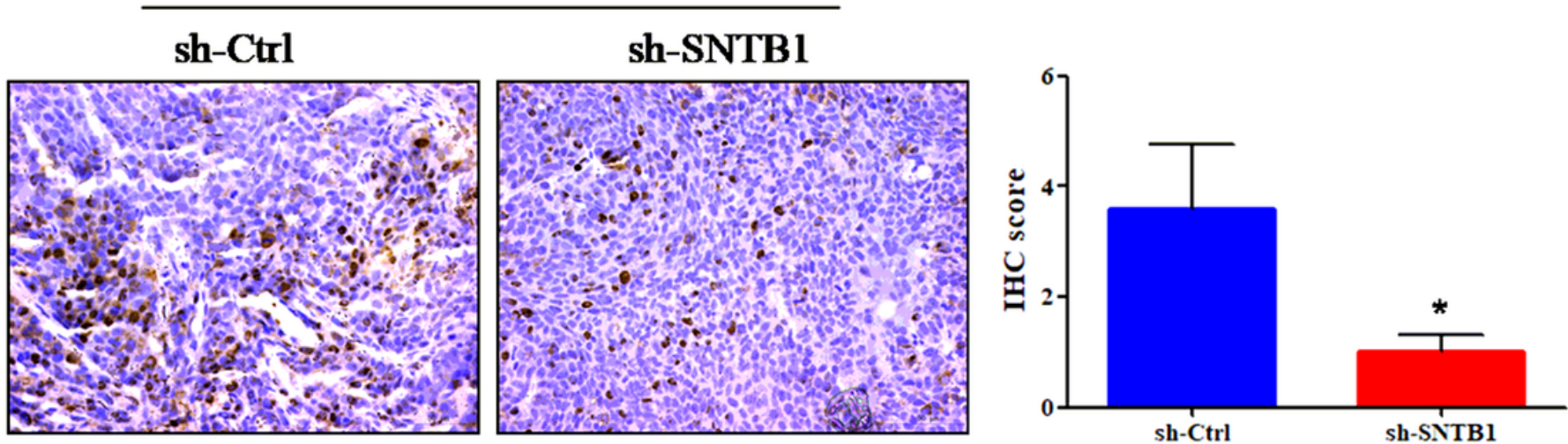

$\mathbf{c}$

TUNEL
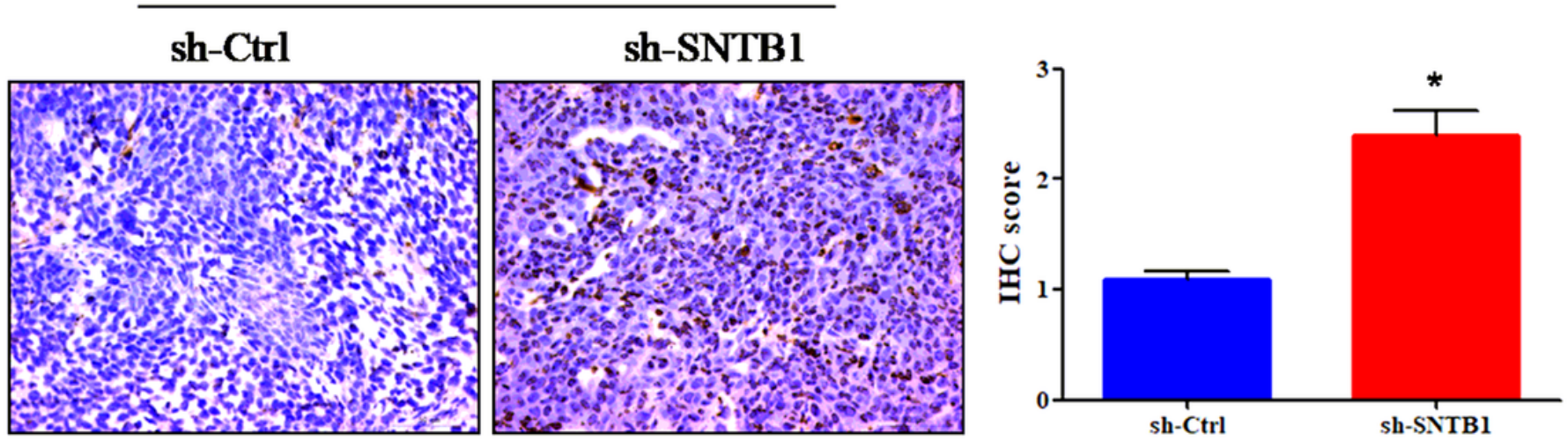

Figure 6

SNTB1 knockdown inhibits cell proliferation and induces cell apoptosis in vivo. IHC was performed to detect SNTB1 (a) and PCNA (b) expression in tumor sections, and TUNEL assay (c) was used to determine the apoptotic cells in tissues of both sh-Ctrl and sh-SNTB1. The representative images of IHC analysis or TUNEL assay were taken at a magnification of $\times 400$ (left panel) and IHC scores were calculated (right panel). $\mathrm{P}<0.05$ vs. sh-Ctrl group). 
a

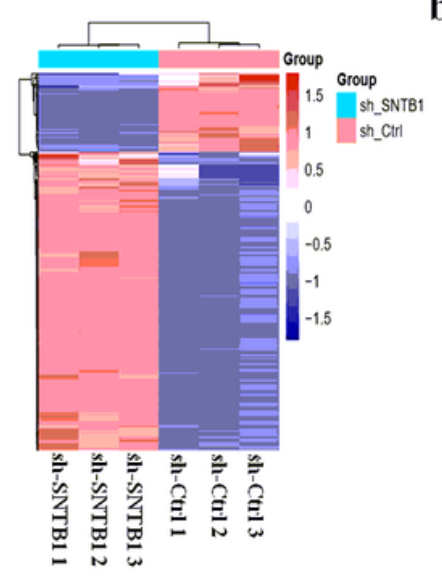

b

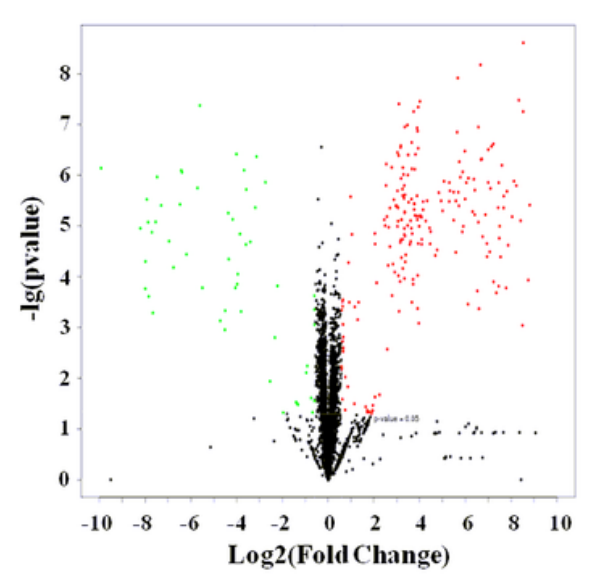

c

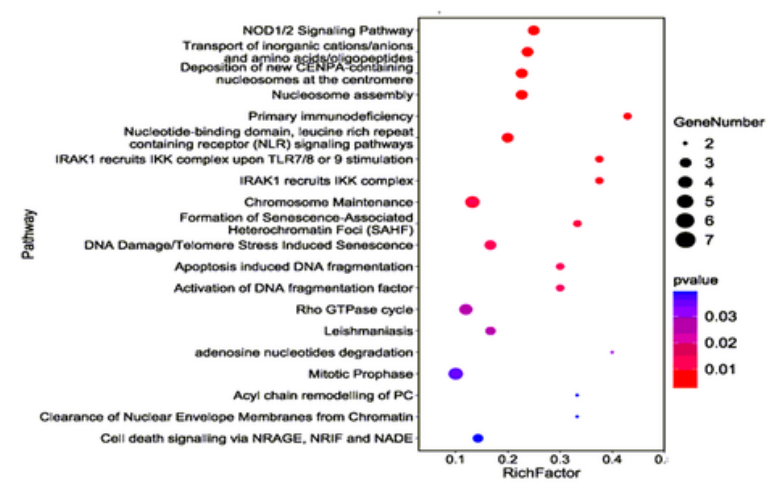

d

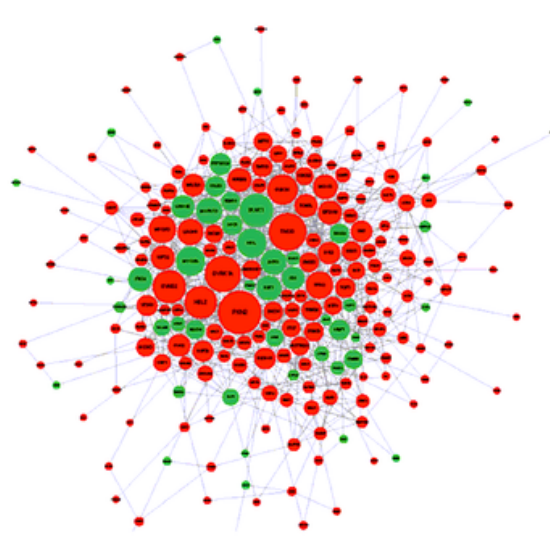

f

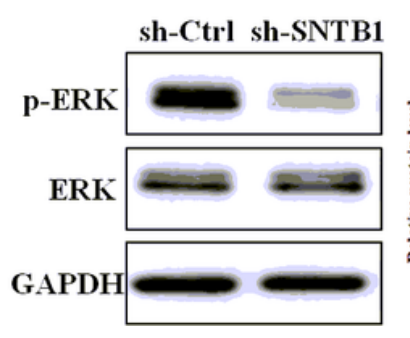

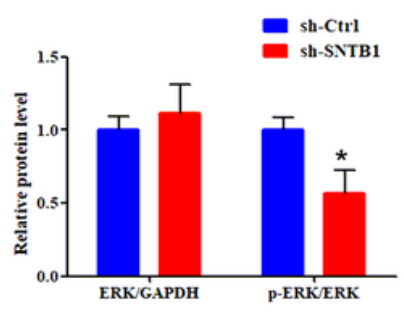

e

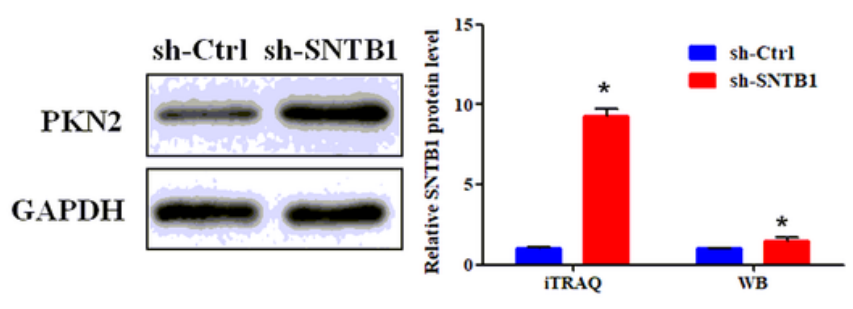

g

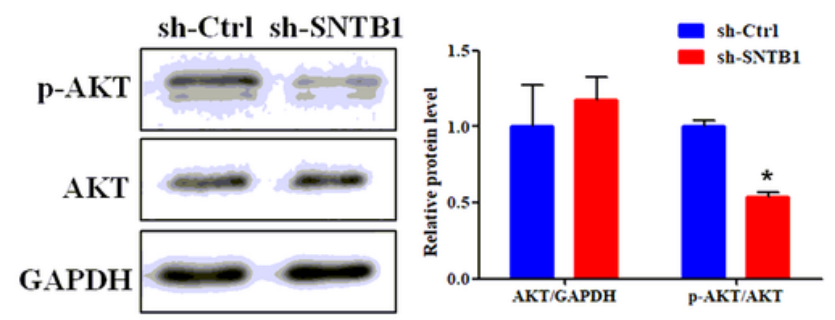

Figure 7

SNTB1 knockdown suppresses PKN2 and activation of ERK and AKT signaling pathway. HCT116 cells were transduced with lentivirus encoding either anti-SNTB1 shRNA (sh-SNTB1) or control shRNA (sh-Ctrl), and ITRAQ was used to identify DEPs. A hierarchical clustering plot (a)and a volcano plot (b) were used to identify DEPs (fold change $\geq 1.5, P<0.05$ ). (c) KEGG pathway enrichment analysis was performed to identify functionally related gene pathways. The top 20 enriched signaling pathways are shown. (d) Protein-protein interaction (PPI) network among DEPs was constructed. Red represent up-regulated proteins, while green represent down-regulated proteins. (e) The protein levels of PKN2 in HCT116 cells after SNTB1 knockdown were determined by Western-blot analysis. The representative images were showed in upper panel and its expression was quantitated using ImageLab software (lower panel). GAPDH was used as an internal control. ${ }^{*} P<0.05$ vs. sh-Ctrl. (f) The protein levels of $p$-ERK and ERK in HCT116 cells after SNTB1 knockdown were determined by Western-blot analysis. The representative images were showed in upper panel and its expression was quantitated using ImageLab software (lower panel). GAPDH was used as an internal control. ${ }^{*} \mathrm{P}<0.05 \mathrm{vs}$. sh-Ctrl. (g) The protein levels of $\mathrm{p}$-AKT and 
AKT in HCT116 cells after SNTB1 knockdown were determined by Western-blot analysis. The representative images were showed in upper panel and its expression was quantitated using ImageLab software (lower panel). GAPDH was used as an internal control. ${ }^{*} \mathrm{P}<0.05 \mathrm{vs}$. sh-Ctrl. All experiments were performed in triplicate.

\section{Supplementary Files}

This is a list of supplementary files associated with this preprint. Click to download.

- supplemnetfigure1.tif

- supplemnetfigure2.tif

- Liuetalsupplementdata.doc 